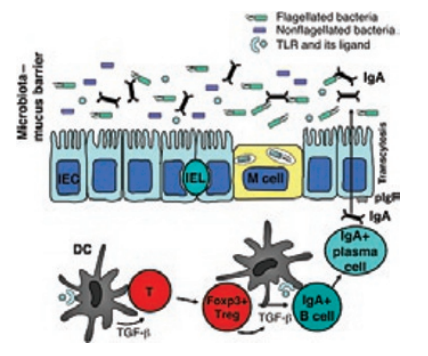

p 15

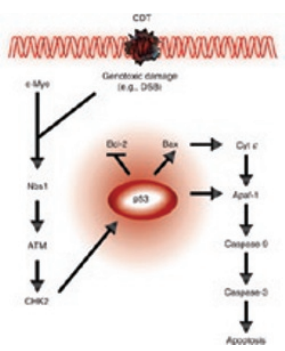

p 22

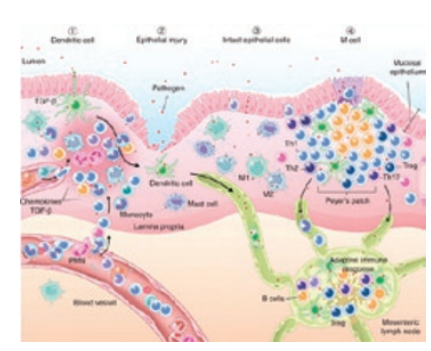

p 31

\section{Fecal transplants}

Alex Khoruts and Michael Sadowsky provide an intriguing commentary on the potential benefits and pitfalls of transplanting distal gut microbiota for the treatment of Clostridium difficile infection. See page 4

\section{Microbial discrimination}

Philippe Sansonetti discusses the discrimination between pathogens and commensal bacteria at mucosal surfaces, a fundamental issue confronting mucosal immunologists. See page 8

\section{Adaptive immunity in host- commensal mutualism}

Ting Feng and Chuck Elson examine the essential role of the adaptive immune system in establishing a mutualistic relationship between commensal bacteria and the host. See page 15

\section{Helicobacter hepaticus}

Jim Fox and colleagues provide a scholarly review of the discovery, immunology, and virulence traits of Helicobacter hepaticus, a cause of chronic liver and colon inflammation as well as colorectal cancer in several mouse models. See page 22

\section{Intestinal macrophages}

Phil Smith and colleagues adeptly address the unique functional phenotype of resident intestinal macrophages and monocytes recruited during infection and inflammation. See page 31

\section{Intestinal bacteria boost airway Treg function}

Deborah Strickland and colleagues demonstrate the ability of gut bacteria to boost baseline regulatory T-cell function as a means of controlling airway hyperresponsiveness in a rat model of asthma. See page 43

\section{Oral bacterial extract inhibits airway inflammation}

Séverine Navarro and colleagues demonstrate that oral administration of Broncho-Vaxom, a commercially available bacterial extract, prevents inflammation in a mouse model of asthma. See page 53

\section{Integrin expression on mucosalT cells}

Seung Kang and colleagues demonstrate coordinated effects of retinoic acid and transforming growth factor- $\beta$ on integrin expression by mucosal homing lymphocytes. See page 66

\section{IL-4 responsiveness of airway smooth muscle cells}

William Horsnell and colleagues describe the contribution of interleukin-4R $\alpha$ responsiveness by smooth muscle cells in driving Thelper type 2-dependent mucus production in the lung during infection with Nippostrongylus brasiliensis. See page 83

\section{Immature thymocytes as precursors for CD8 $\alpha \alpha$ IELs}

Studies by Laetitia Peaudecerf and colleagues indicate that a population of very immature $\mathrm{CD} 44^{+}$thymocytes can give rise to $C D 8 \alpha \alpha$ intraepithelial lymphocytes in the intestine. See page 93

\section{Secretory IL-1 receptor antagonist}

Frederic Carvalho and colleagues demonstrate a critical role for Toll-like receptor-5-dependent secretory interleukin-1 receptor antagonist secretion by epithelial cells in controlling intestinal inflammation. See page 102

\section{HLA-gliadin peptide chimeric proteins}

Jianya Huan and colleagues describe the ability of single-chain recombinant HLA-DQ2.5-gliadin peptide chimeric proteins to inhibit the responses of $\mathrm{T}$ cells in patients with celiac disease. See page 112 